\title{
Sample-displacement correction for whole-pattern profile fitting of powder diffraction data collected in capillary geometry
}

\author{
Nicola Vivienne Yorke Scarlett, Matthew R. Rowles, Kia S. Wallwork and \\ lan C. Madsen
}

J. Appl. Cryst. (2011). 44, 60-64

\begin{tabular}{|l|}
\hline IUCr Journals \\
CRYSTALLOGRAPHY JOURNALS ONLINE \\
Copyright C International Union of Crystallography \\
Author(s) of this paper may load this reprint on their own web site or institutional repository provided that \\
this cover page is retained. Republication of this article or its storage in electronic databases other than as \\
specified above is not permitted without prior permission in writing from the IUCr. \\
For further information see http://journals.iucr.org/services/authorrights.html
\end{tabular}


Journal of

Applied

Crystallography

ISSN 0021-8898

Received 2 November 2010

Accepted 16 November 2010

\section{Sample-displacement correction for whole-pattern profile fitting of powder diffraction data collected in capillary geometry}

\author{
Nicola Vivienne Yorke Scarlett, ${ }^{a *}$ Matthew R. Rowles, ${ }^{a}$ Kia S. Wallwork ${ }^{\mathrm{b}}$ and Ian C. \\ Madsen $^{\mathrm{a}}$ \\ aprocess Science and Engineering, CSIRO, Box 312, Clayton South, Victoria 3169, Australia, and \\ b Australian Synchrotron, Clayton, Victoria 3168, Australia. Correspondence e-mail: \\ nicola.scarlett@csiro.au
}

\begin{abstract}
Recent in situ experimentation at the Australian Synchrotron resulted in the nucleation and crystallization of material on the walls of the capillary reaction vessels. This lining of the capillary walls, without filling the bulk of the capillary volume, produced an artefact in the diffraction data due to sample displacement across the capillary. In effect, the experiment was examining simultaneously two samples displaced by equal and opposite amounts from the diffractometer centre. This was exaggerated by the fact that large-diameter $(1 \mathrm{~mm})$ capillaries had been used in order to increase the total sample volume and hence maximize the amount of material formed and examined. The effect of this displacement was to shift the diffraction peaks simultaneously to both lower and higher angles than their 'ideal' positions, causing peak splitting in many instances. A model has been developed which considers the sample as being effectively two flat plate samples, thus allowing for correction through the use of sample displacement. An additional problem resulted from the oriented growth of the material on the capillary walls, producing preferred orientation in the observed data. The correction model can also be extended to model such anisotropic peak splitting caused by this preferential orientation of the crystallites on the capillary wall.
\end{abstract}

(C) 2011 International Union of Crystallography Printed in Singapore - all rights reserved

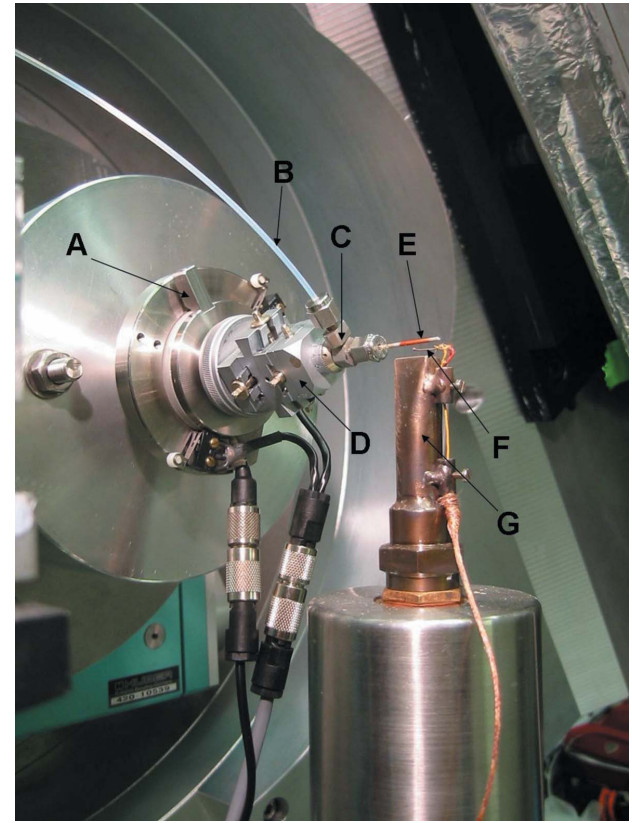

Figure 1

The sample environment for the in situ crystallization experiments, showing (A) the oscillation device for the sample stage, (B) the pressure line, (C) the Swagelock sample stage, (D) the goniometer head holding the sample stage, (E) the capillary reaction vessel, $(\mathrm{F})$ the thermocouple and $(\mathrm{G})$ the hot-air blower. 
Fig. 3. In order to model adequately the structure of the material generating the diffraction pattern, this sampledisplacement artefact must be described in order to separate it from any physical or chemical parameters, which may then be refined. This has been achieved by considering the experimental geometry and has been tested by preparing a series of samples that exhibit this displacement in a controlled fashion.
Figure 2

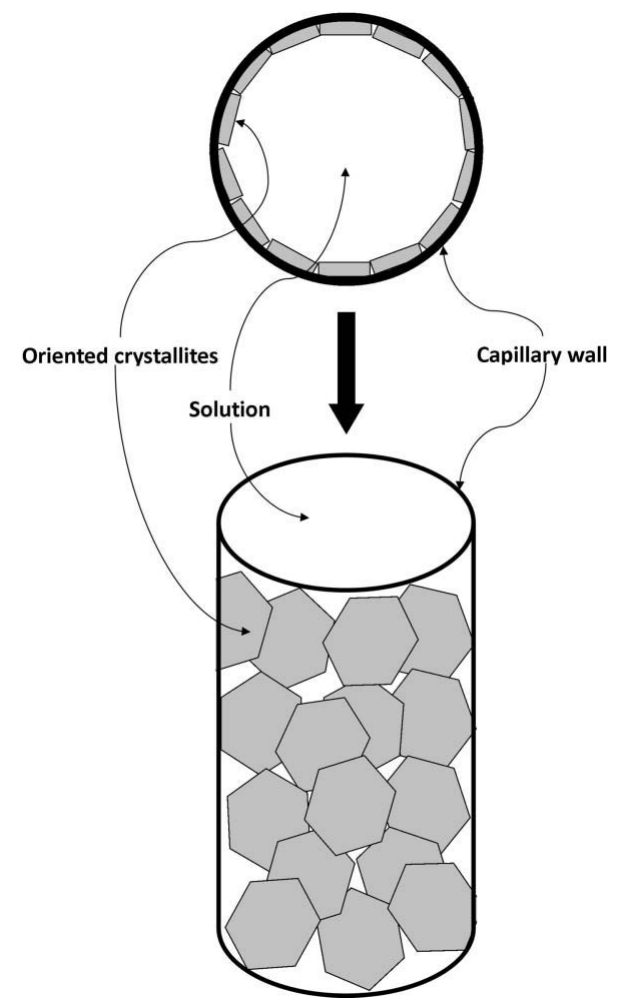

Schematic diagram showing a capillary reaction vessel with oriented crystallites lining its internal wall.

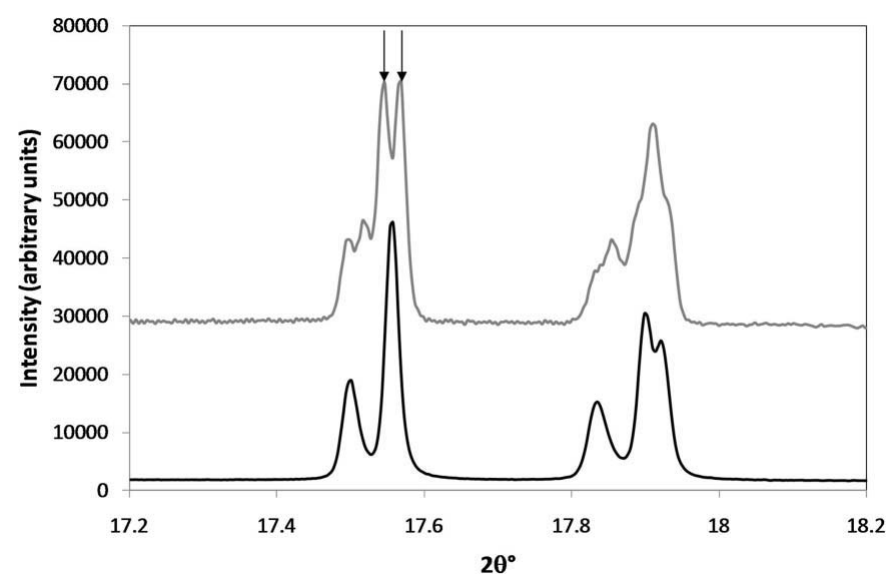

Figure 3

Parts of two diffraction patterns for natrojarosite, collected ex situ in a $0.3 \mathrm{~mm}$ capillary (black, lower), and in situ in a $1 \mathrm{~mm}$ capillary (grey, upper, offset for clarity) prepared at $368 \mathrm{~K}$. An example of the peak splitting due to sample displacement across the capillary is indicated by the arrows.

\section{Experimental}

\subsection{Model development}

Fig. 4 shows how the position of the diffracted beam shifts as a result of sample displacement either $(a)$ parallel or $(b)$ perpendicular to a parallel incident beam in Debye-Scherrer geometry. The sample, $S$, is displaced from the centre of the diffractometer, $O$, by a distance $u$. These displacements shift the diffracted peak from $O^{\prime}$ to $S^{\prime}$, with apparent diffraction angles of $2 \theta-\alpha$ for a parallel displacement and $2 \theta+\beta$ for a perpendicular displacement. The angles $\alpha$ and $\beta$ are given by

$$
\sin \alpha=\frac{u}{R} \sin (2 \theta)
$$

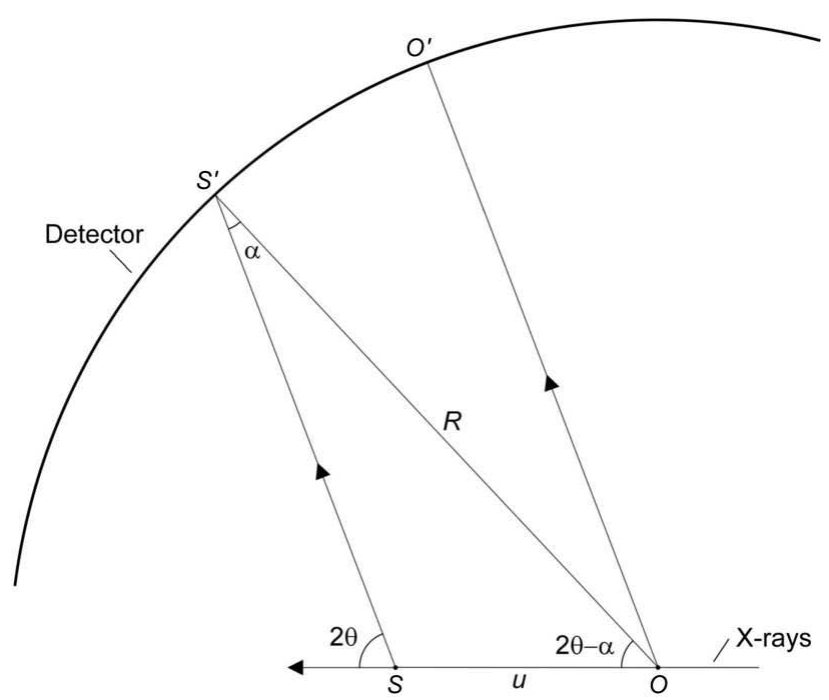

(a)

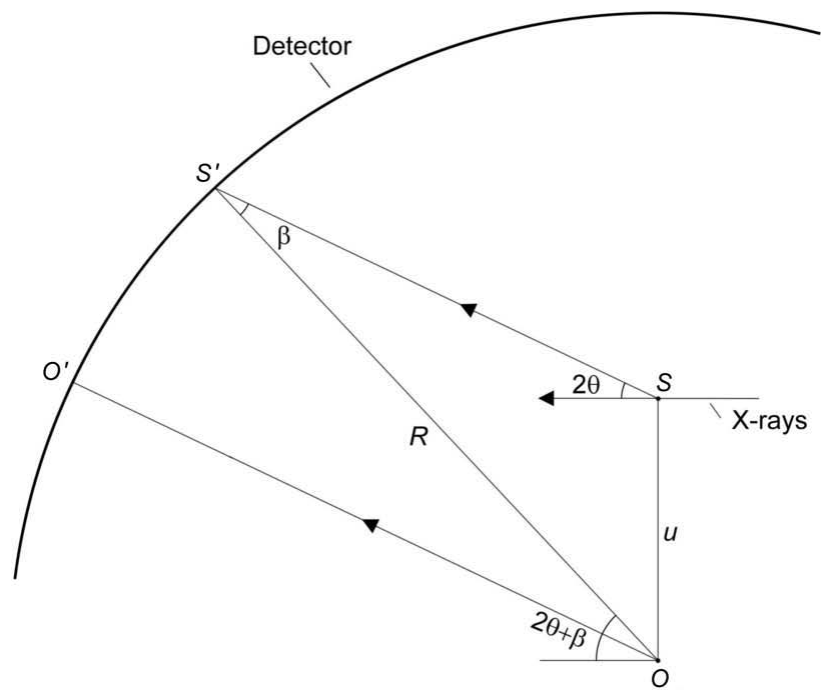

(b)

Figure 4

Peak shift due to sample displacement in Debye-Scherrer geometry. The sample, $S$, can be displaced from the origin, $O$, in a direction either $(a)$ parallel or $(b)$ perpendicular to the incident beam, resulting in the diffraction peak shifting from $O^{\prime}$ to $S^{\prime}$. The effective diffraction angles are $2 \theta-\alpha$ for a parallel displacement and $2 \theta+\beta$ for a perpendicular displacement. The detector radius is given by $R$. 


$$
\sin \beta=\frac{u}{R} \cos (2 \theta)
$$

where $2 \theta$ is the diffraction angle, $u$ the sample displacement and $R$ the detector radius. Equations (1) and (2) are embodied in the correction for sample eccentricity given by Klug \& Alexander (1974) and modified from Beu \& Scott (1962), given a parallel incident beam. Parallel and perpendicular displacements can be applied simultaneously to provide offaxis displacement corrections.

In the situation of having crystallization on the surface of a capillary, and a parallel incident X-ray beam smaller than the diameter of the capillary, it is only necessary to correct for sample displacement both in front of and behind the sample centre. This is achieved by modelling the data with two identical structures, one with a positive and one with a negative parallel displacement, i.e the measured peak positions are corrected using equation (1), with equal and opposite displacements of half the capillary diameter. Note that the cylindrical nature of the sample is not being modelled, the sample is being considered as effectively two flat plates and a well aligned diffractometer is assumed. In the crystallization experiments considered in this study, absorption was found to have a negligible effect on peak intensity and position and was not considered further. For a discussion of X-ray absorption in annular samples, please refer to Kendig \& Pings (1965).

\subsection{Sample preparation}

In order to test this model, samples were prepared in which a material prone to orientation [brucite, $\mathrm{Mg}(\mathrm{OH})_{2}$; Fig. 5] was coated onto the outside of capillaries of various diameters (nominally $0.3,0.5,1.0$ and $1.5 \mathrm{~mm}$ ). This was achieved by repeatedly dipping the capillaries into a stirred slurry of brucite in $\mathrm{H}_{2} \mathrm{O}$, to which a few drops of cellulose lacquer had been added to assist with adhesion. The structural parameters of brucite were initially determined from a data set collected

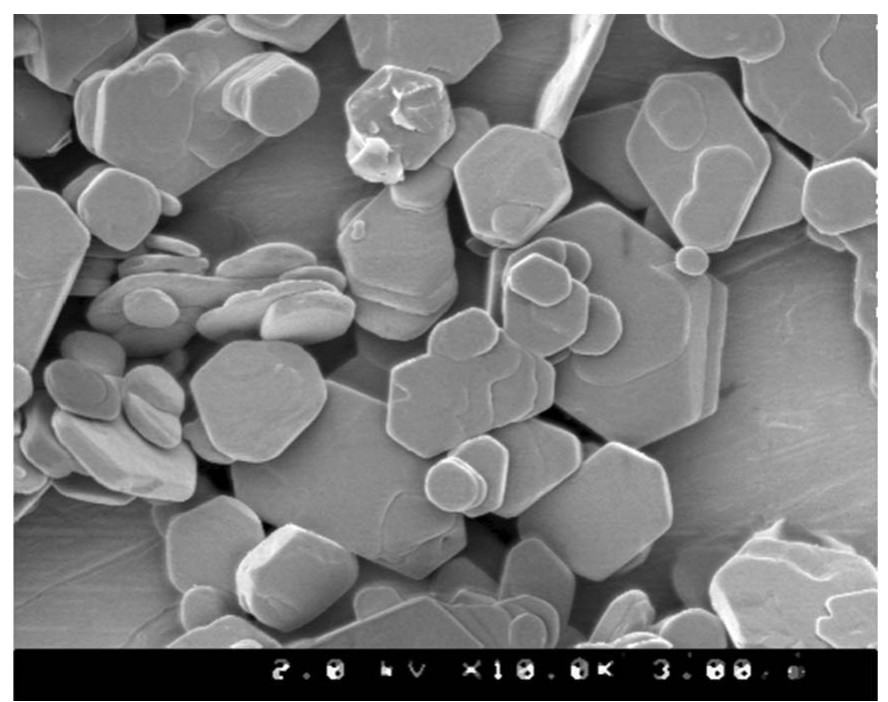

Figure 5

Brucite morphology, showing the flat hexagonal plates which lead to preferred orientation along the [001] direction. from a filled $0.3 \mathrm{~mm}$ capillary and then fixed in the subsequent refinement of the data from the coated capillaries. The model was also applied to diffraction data collected from jarosite samples formed in situ as described above.

\subsection{Data collection}

Data were collected using $0.953421 \AA$ X-rays, calibrated to NIST standard $\mathrm{LaB}_{6} 660 \mathrm{a}$, from samples coated onto capillaries of nominal diameter $0.3,0.5,1.0$ and $1.5 \mathrm{~mm}$, as described above. In order to maximize peak splitting resulting from crystallization on the walls of the capillaries, the vertical size (equatorial plane) of the X-ray beam was set such that it was only just larger than a $0.3 \mathrm{~mm}$-diameter capillary. In doing so, it was also possible to acquire data from the ideal case - a filled capillary - without aberrations induced by the instrument geometry. This allowed comparison of the ideal data with data from samples that were larger in diameter than the vertical beam size. All capillaries were positioned in the

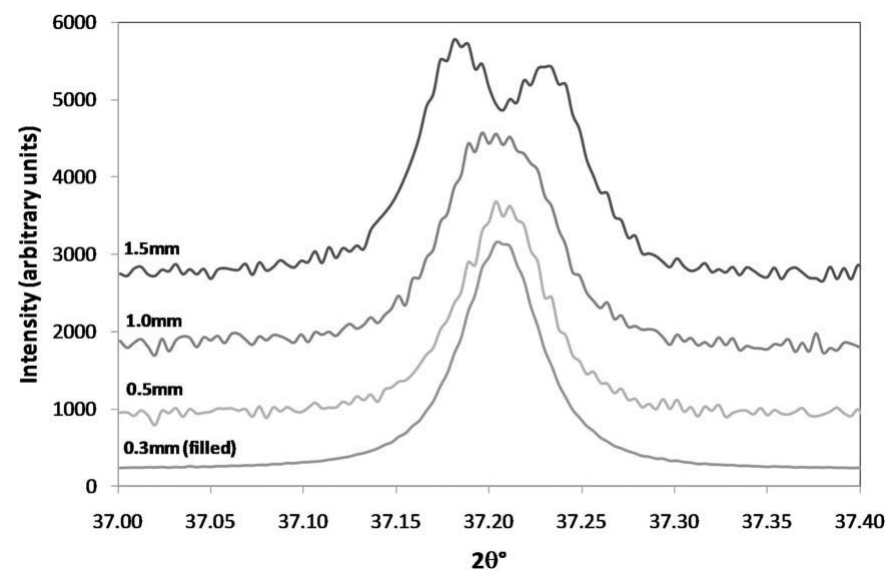

Figure 6

The 111 reflection of brucite, collected from samples coated onto 1.5, 1.0 and $0.5 \mathrm{~mm}$ capillaries and one $0.3 \mathrm{~mm}$ capillary packed with powder, showing the evolution of peak splitting with sample displacement.

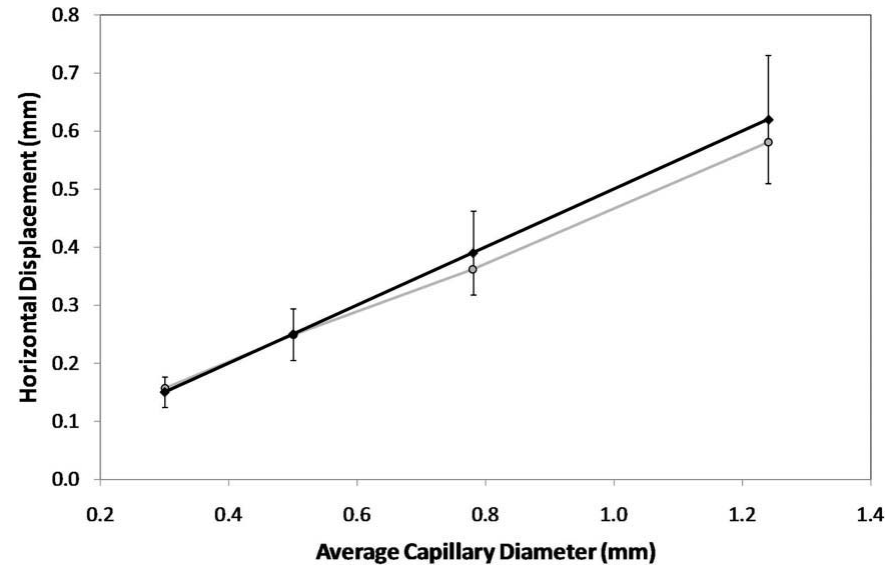

Figure 7

A graph showing the refined values of the horizontal specimen displacement for each capillary diameter (black) compared with the theoretical values (i.e. half the capillary diameter) (grey). The error bars represent the standard deviation in the measured capillary diameter. 
diffractometer rotation centre and spun at $\mathrm{ca} 1 \mathrm{~Hz}$. The X-ray beam was aligned to coincide with the diffractometer centre.

\section{Results and discussion}

Fig. 6 shows the peak broadening and eventual splitting of the brucite (111) reflection associated with specimen displacement. Full-profile Rietveld refinement using TOPAS (Bruker, 2009) was applied to each data set, using the structural information of Černý et al. (1995) for brucite. A model was constructed in which the structural parameters (unit-cell parameters, thermal displacement parameters) were fixed to the values determined from the data set measured on the filled $0.3 \mathrm{~mm}$ capillary. It was found that the sample had oriented preferentially on the capillaries through the sample-prepara-
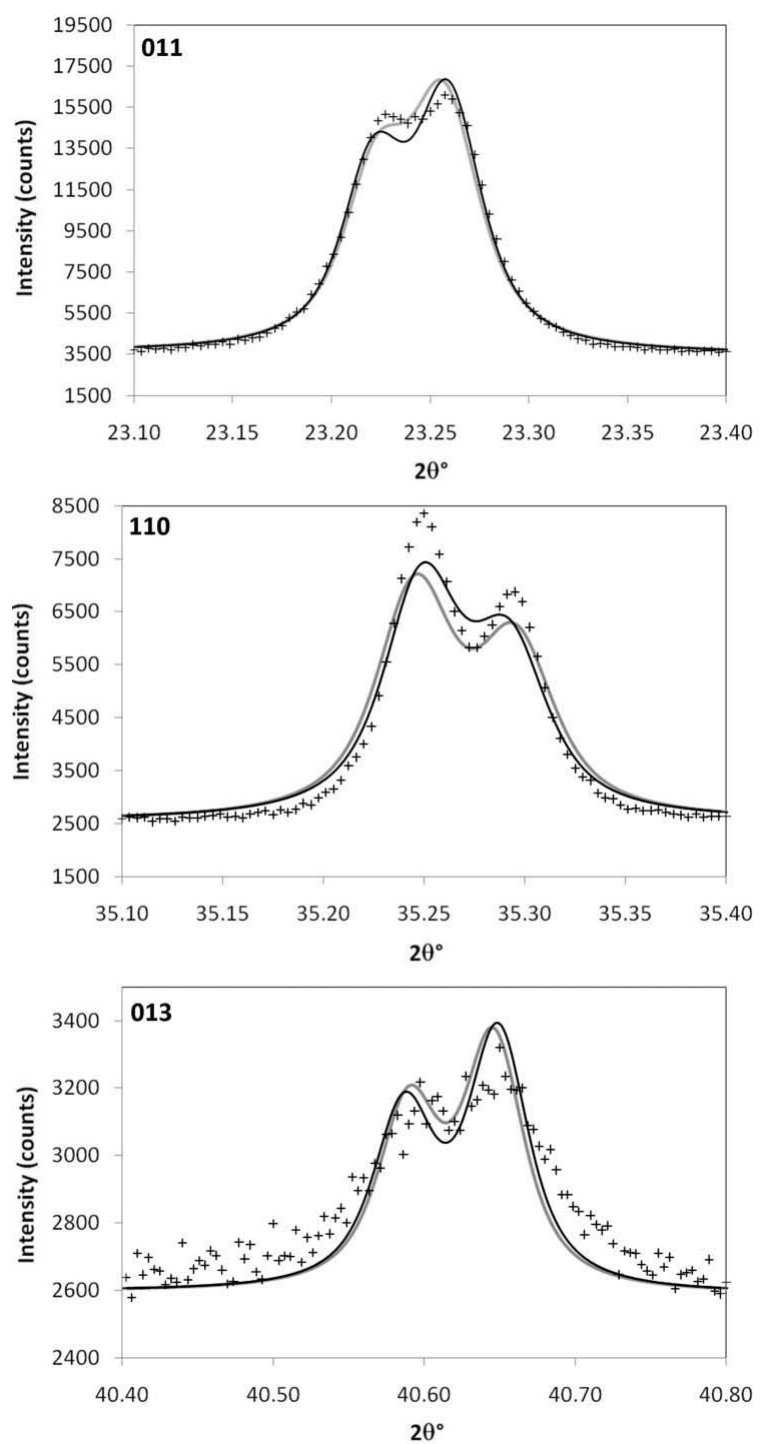

Figure 8

Parts of the Rietveld-fitted diffraction patterns for brucite coated onto a $1.5 \mathrm{~mm}$ capillary. The symbols are the experimental points and the lines correspond to the calculated patterns. The grey lines correspond to a model for which a single (equal and opposite) horizontal displacement value has been refined, and the black lines show the results of the reflection-dependent displacement correction. tion (dipping) process. It was necessary to model this unusual sample orientation using spherical harmonics for the data collected from the coated capillaries. The sample displacement was modelled using two identical structures, with their scale factors constrained to be equal and with equal and opposite horizontal displacement values. Fig. 7 shows the refined values for the specimen-displacement parameters for each capillary diameter (black), along with the theoretical values (grey), which are equal to half the capillary diameter if the capillary is perfectly centred. These are in very good agreement, considering the variation in capillary diameter from the nominal manufactured value and any potential misalignment of the capillaries.

Given the highly oriented nature of the brucite, a reflectiondependent version of the sample-displacement correction was applied by allowing each class of reflections to have an independent sample-displacement parameter. This provided a slight improvement in the most extreme case $(1.5 \mathrm{~mm}$ capillary), with a reduction in $R_{\text {wp }}$ from 0.02075 to 0.01997 . However, as can be seen in Fig. 8, the improvement in the fit is barely discernible and does not warrant the added degree of complexity.
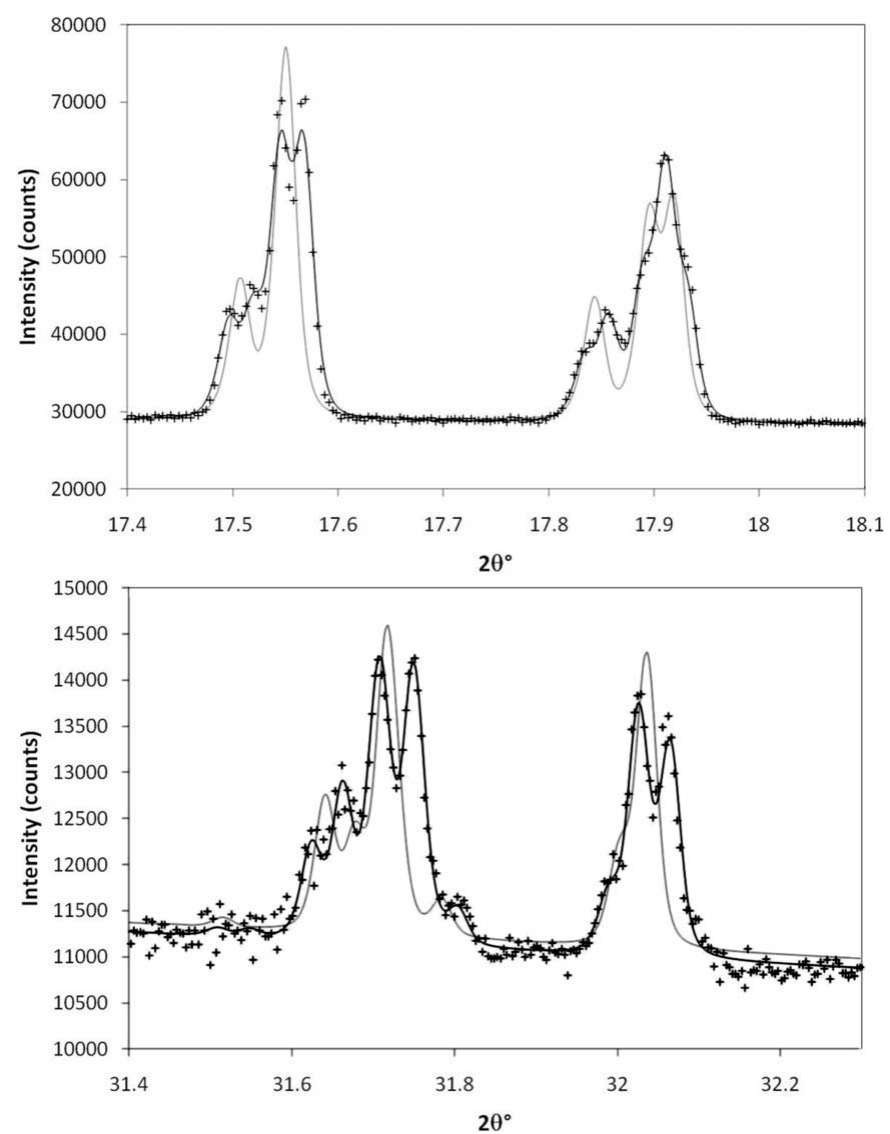

Figure 9

Parts of the Rietveld-fitted diffraction patterns for natrojarosite formed in situ in a $1 \mathrm{~mm}$ capillary at $368 \mathrm{~K}$. The symbols are the experimental points and the lines correspond to the calculated patterns. The grey lines correspond to a model for which the horizontal displacement is not corrected, and the black lines show the results of the displacement correction. 
Fig. 9 shows parts of the Rietveld-fitted diffraction patterns for natrojarosite formed in situ in a $1 \mathrm{~mm}$ capillary at $368 \mathrm{~K}$ (Scarlett et al., 2010). The model was initially refined with a single monoclinic natrojarosite structure and no correction for horizontal displacement (grey lines). It was then modified to include a second, identical, structure with equal and opposite displacement parameters, as described for the brucite case above (black lines). The improvement in the fit to the observed data is clearly apparent and the $R_{\mathrm{wp}}$ value reduced from 0.02667 to 0.01287 . Note that these values are inherently low, as a result of the large contribution to the background from the solution inside the capillary. The refined value of the displacement parameter was $0.499 \mathrm{~mm}$, which is consistent with a $1 \mathrm{~mm}$ capillary reaction vessel.

\section{Conclusion}

A sample-displacement correction has been developed to deal with unusual sample presentations found in crystallization studies. The correction addresses the issue of Debye-Scherrer geometry in which the capillary is not actually filled with sample, but rather coated with crystallites either internally or externally. The correction considers the sample as comprising two separate samples equally displaced about the diffractometer centre in the direction of the incident X-ray beam. The method has been tested with a series of samples that exhibit this displacement in a controlled fashion and also on a sample produced during an in situ crystallization study, and found to be effective. The method can be modified to be made reflection-dependent to deal more accurately with preferential orientation of crystallites, but this was found to be unnecessary in the cases studied here.

This research was undertaken on the powder diffraction beamline at the Australian Synchrotron, Victoria, Australia. The authors express their thanks to Ian Grey, Helen Brand, Suzanne Neville and Nathan Webster (CSIRO Process Science and Engineering) for help with the synchrotron data collections.

\section{References}

Beu, K. E. \& Scott, D. L. (1962). Acta Cryst. 15, 1301-1304.

Bruker (2009). TOPAS. Version 4.2. Bruker AXS Inc., Madison, Wisconsin, USA.

Černý, R., Valvoda, V. \& Chládek, M. (1995). J. Appl. Cryst. 28, $247-$ 253.

Kendig, A. P. \& Pings, C. J. (1965). J. Appl. Phys. 36, 1692-1698.

Klug, H. P. \& Alexander, L. E. (1974). X-ray Diffraction Procedures for Polycrystalline and Amorphous Materials, 2nd ed. New York: Wiley.

Scarlett, N. V. Y., Grey, I. E. \& Brand, H. E. A. (2010). Am. Mineral. 95, 1590-1593.

Scarlett, N. V. Y., Madsen, I. C. \& Whittington, B. I. (2008). J. Appl. Cryst. 41, 572-583. 